\title{
Pneumonia in Indiana nursing homes: a retrospective case series
}

\author{
Andrew Shearn ${ }^{1}$, Kathleen Unroe, MD, $\mathrm{MHA}^{1,2,3}$, Jennifer Carnahan, MD, MPH, \\ $\mathrm{MA}^{1,2,3}$ \\ ${ }^{1}$ Indiana University School of Medicine, ${ }^{2}$ Regenstrief Institute, Inc., \\ ${ }^{3}$ Indiana University Center for Aging Research
}

\section{Background}

The Optimizing Patient Transfers, Impacting Medical Quality, \& Improving Symptoms: Transforming Institutional Care (OPTIMISTIC) project is a Centers for Medicare and Medicaid (CMS) demonstration project, tasked with reducing potentially avoidable hospitalizations of nursing home residents. OPTIMISTICenrolled nursing homes are reimbursed by CMS for treating residents with pneumonia in place. The purpose of this study is to examine the diagnosis, treatment, and outcomes of episodes of pneumonia in OPTIMISTIC nursing homes.

\section{Project Methods}

This case series uses data from nursing home medical records of the seven facilities with the highest pneumonia caseload identified from the OPTIMISTIC database. Cases are from billing episodes spanning November 2017 through April 2018. Within each facility, cases of pneumonia were randomly selected for inclusion. Data were entered into an extraction tool designed by the study team.

\section{Results}

Data were extracted from 41 records of unique patients. Despite CMS reimbursing for a maximum of 7 days for treatment of pneumonia, $78.0 \%$ of patients were monitored beyond that time and with greater attention than usual care. Of all 41 patients treated with antibiotics, $53.7 \%$ were given a fluoroquinolone and $24.4 \%$ were given amoxicillin/clavulanate. CURB-65 scores showed $58.3 \%$ scored in a range recommending hospitalization. Most patients $(87.8 \%)$ were stabilized in the nursing home; three $(7.3 \%)$ were hospitalized, one $(2.4 \%)$ transferred to hospice, and one $(2.4 \%)$ died.

\section{Conclusion and Potential Impact}

OPTIMISTIC-affiliated nursing facilities successfully provide enhanced care for most patients diagnosed with pneumonia in the facilities. Given the high incidence of fluoroquinolone use, one area for improvement is reduction of this medication contraindicated in the elderly. 